\title{
ОБРАЗОВАНИЕ ФОТОИНДУЦИРОВАННОГО АНТИПРОВАЛА В НЕОДНОРОДНЫХ ПРИМЕСНЫХ СПЕКТРАХ
}

\author{
(Представил К. К. Ребане)
}

1. Фотохимическое выжигание провалов в неоднородных примесных спектрах $\left[{ }^{1-3}\right]$ основано на селективном фотоиндуцировании химических превращений, приводящих к перераспределению примесей по частоте перехода. В недавних работах $\left[{ }^{4,5}\right]$ показано, что при одновременном широкополосном возбуждении центров и спектрально-селективном светоиндуцированном опустошении возбужденного уровня, с которого идут фотохимические превращения, в неоднородно уширенном спектре возникает узкий антипровал. В отличие от выжигания провала образование антипровала связано с фотохимическими превращениями всех образующих неоднородно уширенную линию центров. В данной работе теоретически рассматривается кинетика происходящей при этом трансформации спектра.

2. Соответствующая механизму образования антипровала $[4,5]$ схема возбуждения примесей и актуальные переходы приведены на рис. 1. Спектрально неселективное заселение $S_{1}\left(0^{\prime}\right)$-состояния осуществляется через возбуждение вибронного состояния $S_{1}\left(1^{\prime}\right)$ и последующую энергетическую релаксацию. Селективное опустошение $S_{1}\left(0^{\prime}\right)$-состояния осуществляется монохроматическим источником на переходе $S_{1}\left(0^{\prime}\right) \rightarrow S_{0}(1)$. Тем самым осуществляется селективное торможение фотохимической реакции при условии неактивности колебания основного состояния в процессе фотореакции. Для описания системы фотовыжи-

Рис. 1. Схема актуальных при образовании антипровала энергетических уровней и переходов. Индексы $A, B, C$ выделяют из сплошного неоднородного распределения три типа центров, отличающихся частотами чисто электронного и вибронного переходов. Прямыми стрелками указано неселективное заселение через вибронные уровни $S_{1}$-состояния $(1,2,3)$ и селективное опустошение $S_{1}$-состояния для центров типа $B$ (стрелка 4 ), волнистыми стрелками - безызлучательная электронно-колебательная релаксация, штриховыми фототрансформация. Спонтанный распад $S_{1}$-состояния не указан.

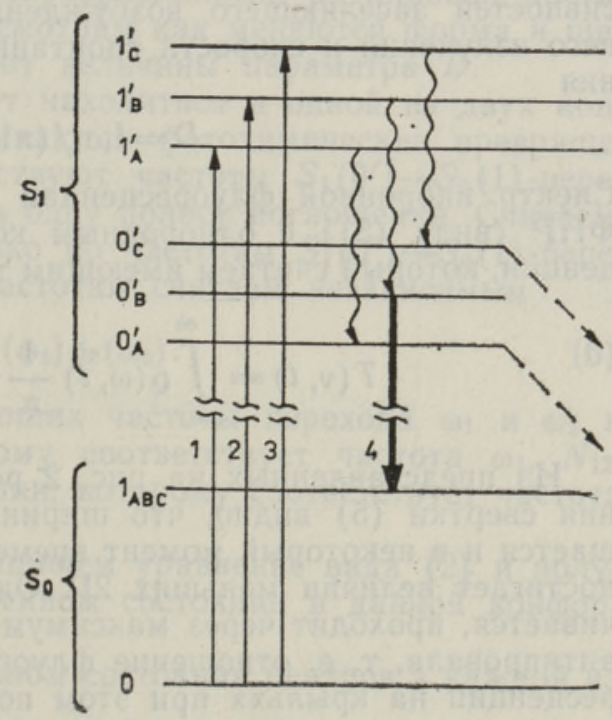


гающихся примесей мы используем модель ([$\left.{ }^{3}\right]$ с. $\left.237-238\right)$, не учитывающую обратные темновые и фотопревращения, взаимодействие между примесями и толщину объекта.

Изменение функции неоднородного распределения (ФНР) примесей по частоте вибронного $S_{1}\left(0^{\prime}\right) \rightarrow S_{0}(1)$-перехода $\varrho(\omega, t)$ в ходе фотореакции описывается уравнением

$$
\partial_{\varrho}(\omega, t) / \partial t=-\eta n(\omega) \varrho(\omega, t),
$$

где $\eta$ - квантовый выход фотохимической реакции, а $n(\omega)-$ доля центров с частотой $S_{1}\left(0^{\prime}\right) \rightarrow S_{0}(1)$-перехода $\omega$, находящихся в возбужденном состоянии. Доля центров, находящихся в основном состоянии, $1-n(\omega)$. Предположим, что фотохимические превращения идут достаточно медленно, для того чтобы между заселенностями уровней установилось равновесие. При равновесии выполняется равенство

$$
I_{0} \sigma_{0}(1-n(\omega))=n(\omega)\left(k+I_{1} \sigma_{1} \frac{1}{\pi(\Gamma / 2)} \frac{(\Gamma / 2)^{2}}{(\Gamma / 2)^{2}+\left(\omega-\omega_{0}\right)^{2}}\right) .
$$

Здесь $I_{0}$ - интегральная интенсивность возбуждающего излучения, $\sigma_{0}$ - интегральное сечение поглощения в основном состоянии для того перехода, с помощью которого равномерно заселяется $S_{1}$-состояние, $k$ - вероятность спонтанного распада $S_{1}$-состояния, $I_{1}-$ интенсивность монохроматического (частота $\omega_{0}$ ) излучения, опустошающего возбужденное $S_{1}$-состояние, $\sigma_{1}-$ интегральное сечение индуцированного сброса заселенности возбужденного состояния, $\Gamma$ - однородная ширина вибронного перехода $S_{1}\left(0^{\prime}\right) \rightarrow S_{0}(1)$, с помощью которого осуществляется селективный сброс заселенности $S_{1}\left(0^{\prime}\right)$-состояния. Соответ-

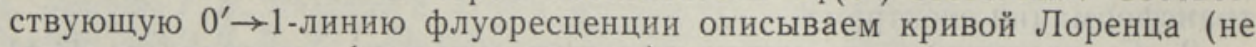
учитывая при этом фононные крылья).

Выразив $n(\omega)$ из (2), подставим его в (1) и получим зависимость ФНР от времени

$$
\begin{gathered}
\varrho(\omega, t)=\varrho_{0}(\omega) \exp \left\{\left(-1+(1-1 /(D+1)) \frac{(D+1) \Gamma^{2}}{(D+1) \Gamma^{2}+\left(\omega-\omega_{0}\right)^{2}}\right) \tilde{t}\right\}, \\
\tilde{t}=I_{0} \sigma_{0} \eta t /\left(I_{0} \sigma_{0}+k\right),
\end{gathered}
$$

где $\varrho_{0}(\omega)$ - ФНР в начальный момент времени (далее везде принимаем $\varrho_{0}(\omega)=$ const), параметр $D$ характеризует соотношение интенсивностей заселяющего возбужденное состояние и сбрасывающего с него излучений и скорости спонтанного распада возбужденного состояния

$$
D=I_{1} \sigma_{1} /\left(\pi \Gamma\left(I_{0} \sigma_{0}+k\right)\right) .
$$

Спектр вибронной флуоресценции центров найдем, вычислив свертку ФНР (вида (3)) с однородным контуром линии вибронной флуоресценции, который считаем имеющим ту же ширину Г

$$
T(v, t)=\int_{-\infty}^{\infty} \varrho(\omega, t) \cdot \frac{1}{\pi} \frac{\Gamma / 2}{(\Gamma / 2)^{2}+(v-\omega)^{2}} d \omega .
$$

Из представленных на рис. 2 результатов численного интегрирования свертки (5) видно, что ширина антипровала со временем уменьшается и в некоторый момент времени, в отличие от обычного провала, достигает величин меньших 2Г. Одновременно высота провала увеличивается, проходит через максимум и начинает уменьшаться (контраст антипровала, т. е. отношение флуоресценции в его максимуме к флуоресценции на крыльях при этом постоянно растет), а при $t \rightarrow \infty$ анти- 


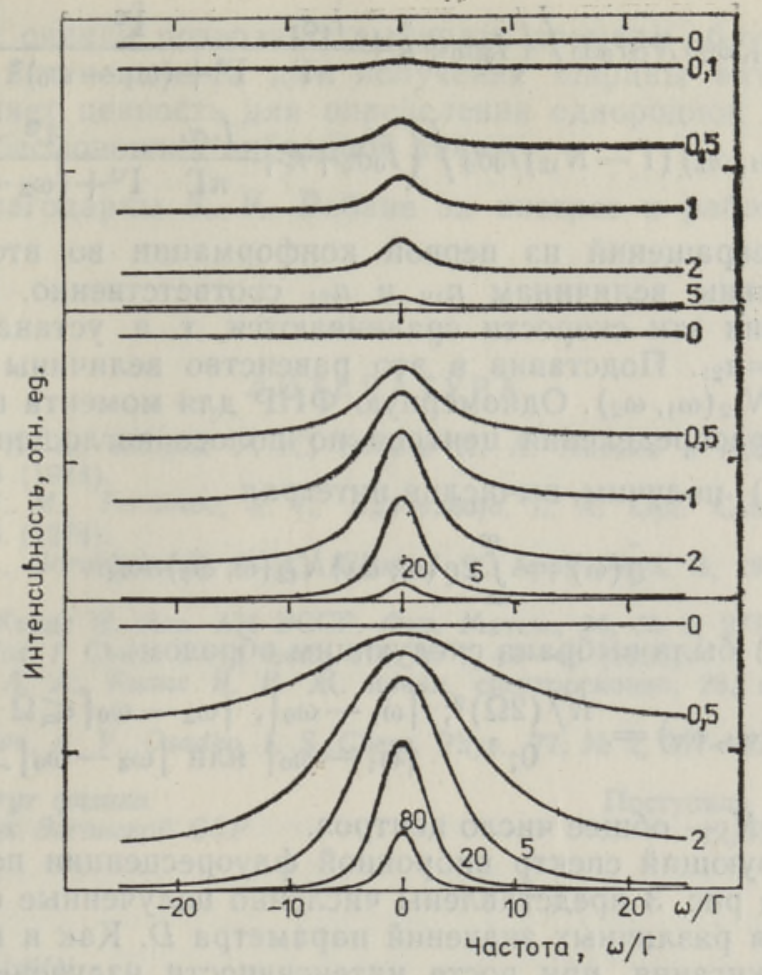

Рис. 2. Временной ход развития антипровала для случаев отсутствия обратных фотохимических превращений. Верхний рисунок соответствует значению $D=1$, средний $D=10$, нижний $D=100$. На правом крыле каждой линии проставлен соответствующий момент времени $\tilde{t}$.

провал исчезает, что означает фотохимическое превращение всех центров, несмотря на торможение реакции. При равных временах облучения, но при различных значениях параметра $D$, увеличение этого параметра ведет к уширению антипровала и увеличению его высоты.

3. В системе с обратными фотопревращениями антипровал достигает насыщения и не исчезает. Рассмотрим как меняются форма и ширина антипровала в зависимости от величины параметра $D$.

Предположим, что центры могут находиться в одной из двух конформаций, между которыми совершаются фотохимические превращения $\left[{ }^{6,7}\right]$. Конформациям соответствуют частоты $S_{1}\left(0^{\prime}\right) \rightarrow S_{0}(1)$-перехода $\omega_{1}$ и $\omega_{2}$ и обе они попадают в одну полосу поглощения. Считаем, что конформации отличаются только по частотам $S_{1}\left(0^{\prime}\right) \rightarrow S_{0}(1)$-перехода. Распределение центров по частотам считаем независимым

$$
\varrho\left(\omega_{1}, \omega_{2}\right)=\varrho_{0}\left(\omega_{1}\right) \varrho_{0}\left(\omega_{2}\right) \text {. }
$$

Обозначим долю центров, имеющих частоты перехода $\omega_{1}$ и $\omega_{2}$ и находящихся в положении, которому соответствует частота $\omega_{1}, N_{12}$, тогда доля находящихся в положении, которому соответствует частота $\omega_{2}: N_{21}=1-N_{12}$.

Для каждой из конформаций запишем уравнение вида (2) и получим долю находящихся в возбужденном состоянии в данной конформации центров.

Число находящихся в возбужденном состоянии центров в каждой из конформаций $n_{12}$ и $n_{21}$ равно: 


$$
\begin{aligned}
& n_{12}=\varrho\left(\omega_{1}, \omega_{2}\right) N_{12} I_{0} \sigma_{0} /\left(I_{0} \sigma_{0}+k+\frac{\dot{I}_{1} \sigma_{1}}{\pi \Gamma} \frac{\Gamma^{2}}{\Gamma^{2}+\left(\omega_{1}-\omega_{0}\right)^{2}}\right), \\
& n_{21}=\varrho\left(\omega_{1}, \omega_{2}\right)\left(1-N_{12}\right) I_{0} \sigma_{0} /\left(I_{0} \sigma_{0}+k+\frac{I_{1} \sigma_{1}}{\pi \Gamma} \frac{\Gamma^{2}}{\Gamma^{2}+\left(\omega_{2}-\omega_{0}\right)^{2}}\right) .
\end{aligned}
$$

Скорости превращений из первой конформации во вторую и назад пропорциональны величинам $n_{12}$ и $n_{21}$ соответственно. При достижении насыщения эти скорости сравниваются, т. е. устанавливается равенство: $n_{12}=n_{21}$. Подставив в это равенство величины (7), получим зависимость $N_{12}\left(\omega_{1}, \omega_{2}\right)$. Одномерную ФНР для момента времени, когда процесс перераспределения центров по полосе поглощения достиг насыщения, $\tilde{\varrho}(\omega)$, получим, вычислив интеграл

$$
\tilde{\varrho}(\omega)=\int_{-\infty}^{\infty} 2 \varrho\left(\omega, \omega_{2}\right) N_{12}\left(\omega, \omega_{2}\right) d \omega_{2} .
$$

ФНР $\varrho\left(\omega_{1}, \omega_{2}\right)$ была выбрана следующим образом:

$$
\varrho\left(\omega_{1}, \omega_{2}\right)=\begin{array}{cl}
N /(2 \Omega)^{2} ; & \left|\omega_{1}-\omega_{0}\right|,\left|\omega_{2}-\omega_{0}\right| \leqslant \Omega \\
0 ; & \left|\omega_{1}-\omega_{0}\right| \text { или }\left|\omega_{2}-\omega_{0}\right|>\Omega,
\end{array}
$$

где $\Omega=100 \Gamma, N$ - общее число центров.

Соответствующий спектр вибронной флуоресценции получим аналогично (5). На рис. 3 представлены численно полученные спектры флуоресценции для различных значений параметра $D$. Как и в случае необратимого выжигания, при росте интенсивности излучения, селективно опустошающего возбужденный уровень относительно интенсивности излучения неселективно его заселяющего, насыщенный антипровал уширяется и увеличивается по высоте. При значениях $D \approx 1$ ширина антипровала $\delta \approx 2 \Gamma$.

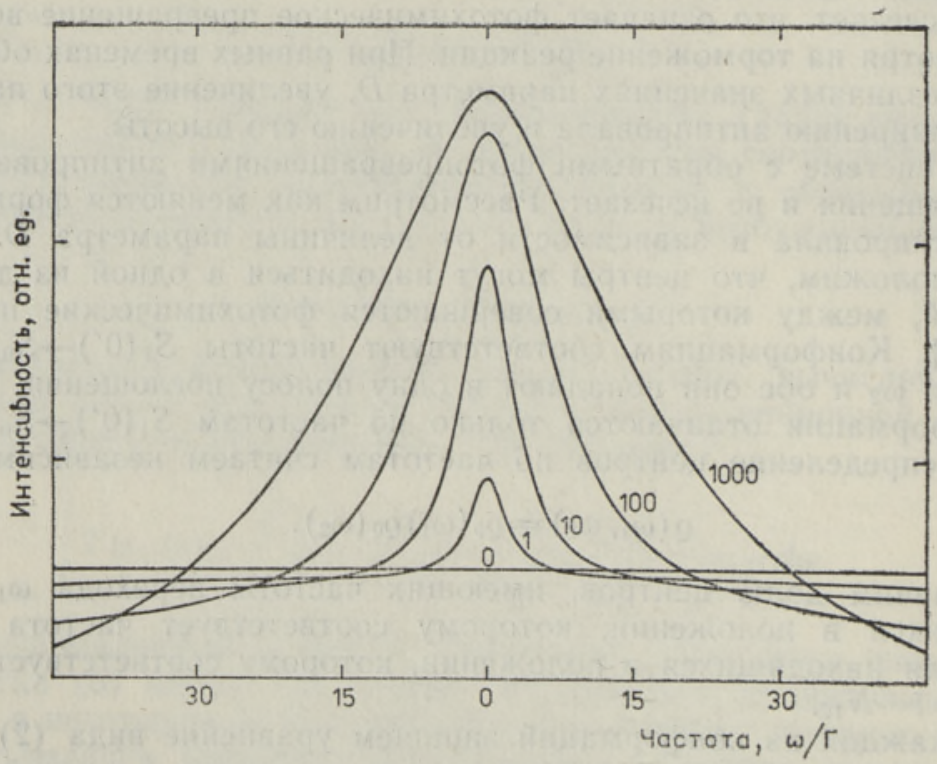

Рис. 3. Насыщенный антипровал, возникающий в системе с примесными центрами, совершающими фотопревращения внутри неоднородно уширенной полосы. Возле кривых проставлено значение параметра $D$. 
Сделанные оценки позволяют выбирать времена облучения и соответствующие интенсивности для получения ширины антипровала $2 \Gamma$, что представляет ценность для определения однородной ширины соответствующей бесфононной вибронной линии.

Авторы благодарны К. К. Ребане за интерес к работе и полезное обсуждение.

\title{
ЛИТЕРА Т У РА
}

1. Гороховский А. А., Каарли Р. К., Ребане Л. А. Письма в ЖЭТФ, 20, вып. 7, $474-479$ (1974).

2. Kharlamov, V. M., Personov, R. I., Bykovskaja, L. A. Opt. Commun., 12, № 1 , 191-193 (1974).

3. Rebane, L. A., Gorokhovskii, A. A., Kikas, J. V. Appl. Phys. B, 29, № 4, 235-250 (1982).

4. Сильдос И., Кикас Я. Изв. АН ЭССР. Физ. Матем., 34, № 3, 271-276 (1985).

5. Kikas, J., Sildos, I. Chem. Phys. Lett., 114, № 1, 44-46 (1985).

6. Гороховский А. А., Кикас Я. В. Ж. прикл. спектроскопни, 28, вып. 5, 832-838 (1978).

7. Jalmukhambetov, A. V., Osadko, I. S. Chem. Phys., 77, № 2, 247-255 (1983).

\author{
Ннститут физики \\ Академии наук Эстонской ССР
}

Поступила в редакцию

12/III 1987

\section{J. KIKAS, J. MALKIN \\ FOTOINDUTSEERITUD ANTISALGU MOODUSTUMINE MITTEHOMOGEENSETES LISANDISPEKTRITES}

Teoreetiliselt on uuritud antisälgu tekke kineetikat mittehomogeenses lisandispektris fotokeemiliselt aktiivse seisundi $S_{1}\left(0^{\prime}\right)$ mitteselektiivsel asustamisel ja selektiivsel tühjendamisel monokromaatse valgusega stimuleeritud $S_{1}\left(0^{\prime}\right) \rightarrow S_{0}(1)$ ülemineku kaudu. Eraldi on vaadeldud juhte, kus puuduvad fotoindutseeritud pöördprotsessid ja kus nimetatud protsessid toimivad. Antisâllgu kuju ja laius sōltuvad oluliselt ergastatud seisundi asustamise ning stimuleeritud ja spontaanse tühjendamise kiiruse vahekorrast. Kiiritamisel antisälk kitseneb.

\section{J. KIKAS, J. MALKIN}

\section{PHOTOINDUCED ANTIHOLE FORMATION IN INHOMOGENEOUS IMPURITY SPECTRA}

Kinetics of photoinduced antihole in inhomogeneous impurity spectra on nonselective population of photochemically active $S_{1}\left(0^{\prime}\right)$-state and its selective depopulation by monochromatic light on the vibronic transition $S_{1}\left(0^{\prime}\right) \rightarrow S_{0}(1)$ is studied theoretically.

The cases of absence and presence of reverse phototransformations are considered. The shape and width of the antihole depend essentially on the rates of population as well as stimulated and spontaneous depopulation of the excited state $S_{1}$. The narrowing of the antihole in time has been noted. 